\title{
POSITRON ANNIHILATION IN DEFORMED AND HYDROGENATED Pd-23\%atCu ALLOY
}

\author{
J. PAJAzK ${ }^{a *}$, R. SzataniK ${ }^{a *}$ AND M. Szuszkiewicz ${ }^{b}$ \\ anstitute of Physics, Opole University, Oleska 48, 45-052 Opole, Poland \\ ${ }^{b}$ Institute of Experimental Physics, University of Wrocław \\ Pl. Maxa Borna 36, 50-205 Wrocław, Poland
}

Peak coincidence counting rate and positron lifetime spectra for deformed by cold-rolling, and annealed by four hours in the temperature $973 \mathrm{~K}$, hydrogen free and hydrogenated $\mathrm{PdCu}$ with $23 \% \mathrm{Cu}$ samples, in the temperature range from room temperature to about $720 \mathrm{~K}$ were measured. It was found that hydrogen improved to tempered samples creates defects that are annealed to the temperature about $525 \mathrm{~K}$. In the cold-rolled up to deformation degree of $50 \%$ samples, hydrogen is trapped at defects, probably vacancies, forming $H-V$ complexes, that become disappearing in the vicinity of the temperature at $350 \mathrm{~K}$.

PACS numbers: $78.70 . \mathrm{Bj}$

\section{Introduction}

Under certain conditions hydrogen could interact with defects in the metals and alloys to form new defects. These new defects further develop and may radically change mechanical properties of constructional materials.

Details of investigations on hydrogen induced defects in metals and alloys are significant for the study of mechanism of hydrogen-induced metal and alloy damages, and for development and application of hydrogen-resistant materials.

This paper presents the data of the investigations on the hydrogen-induced defects as well as the interaction between hydrogen and defects in the disordered $\alpha$ phase of PdCu alloy.

The measurements of the changes in the coincidence peak counting rate as a function of temperature and positron lifetime for the annealed and cold-rolling-deformed hydrogen-free and hydrogen-saturated $\mathrm{Pd}-23 \% \mathrm{atCu}$ samples are presented.

\section{Experimental details}

The $\mathrm{PdCu}$ alloy with $23 \%$ at. $\mathrm{Cu}$ crystallizes in fcc structure forming disordered $\alpha$ phase. More detailed information can be found in [1]. The samples with dimensions $14 \times 12 \times 1 \mathrm{~mm}^{3}$ were annealed for four hours at $973 \mathrm{~K}$ and slowly quenched up to room temperature. Afterwards some of the samples were deformed by cold-rolling up to deformation degree of $50 \%$. The deformed and an-

*e-mail: chabik@uni.opole.pl 
nealed samples were electrically charged with hydrogen to concentration of about $2 \times 10^{-2} \mathrm{atH} /$ atPdCu in $1 \mathrm{~N}$ aqueous solution of $\mathrm{H}_{2} \mathrm{SO}_{4}$. The peak coincidence counting rate for both annealed and deformed hydrogen-free and hydrogenated samples were measured in the temperature range from room temperature to $720 \mathrm{~K}$, using the standard correlation spectrometer with a long-slit geometry. The positron lifetime spectra for the series of the samples were obtained at room temperature using a conventional fast-slow coincidence setup with $\mathrm{BaF}_{2}$ scintillators and time resolution (FWHM) about 260 ps.

\section{Results and discussion}

In Fig. 1 the normalized coincidence peak counting rate as a function of the temperature for the annealed hydrogen-free $\mathrm{PdCu}$ alloy sample (black squares) and hydrogenated one (open circles) are shown. As shown in Fig. 1, the curve for hydrogenated sample may be divided into three parts. The first part is in the range of the temperature from room temperature to about $525 \mathrm{~K}$. The second part begins at the temperature about $375 \mathrm{~K}$ and ends about $525 \mathrm{~K}$. The last part, common for two curves for hydrogen-free and hydrogenated samples is in the range of the temperature above $525 \mathrm{~K}$.

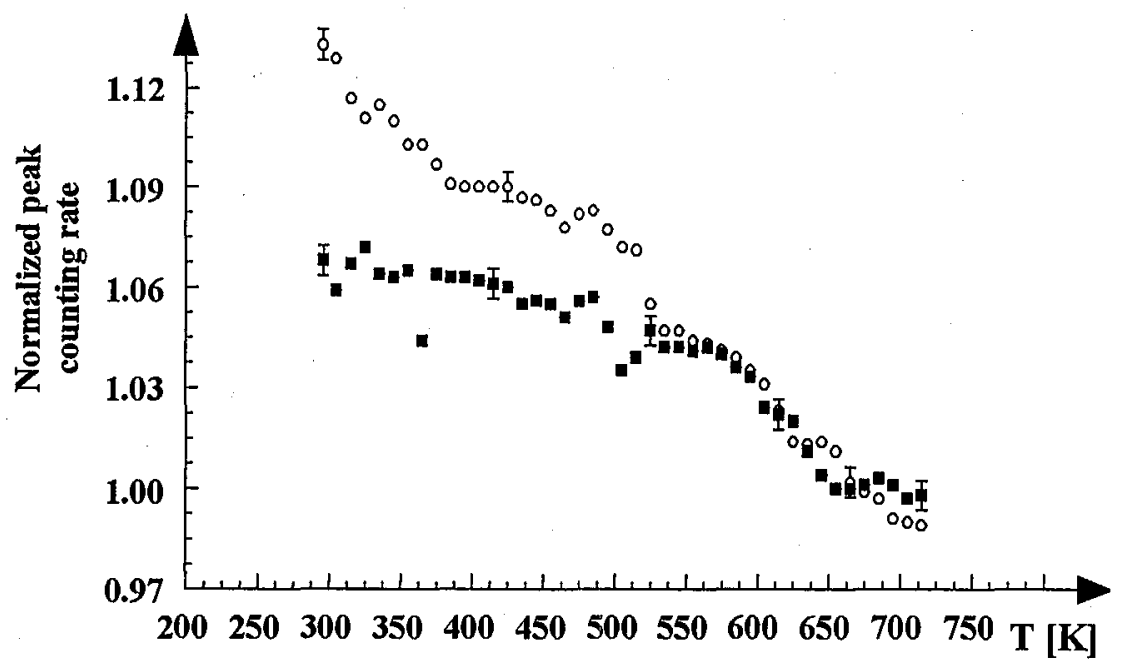

Fig. 1. Normalized peak counting rate as a function of the temperature for annealed $\mathrm{Pd}-23 \% \mathrm{Cu}$ sample (black squares), and for annealed and hydrogenated sample (open circles).

The first part of the curve, most sharply dropping, may be explained as a result of hydrogen-induced defects, probably hydrogen vacancy complexes [2] that are annealed or migrate to form more stable three-dimensional H-defect systems.

These systems are stable up to the temperature of about $500 \mathrm{~K}$, i.e. up to the temperature at which hydrogen atoms become very mobile [3] (the second part of the curve). The third part of the curve is due to the recrystallization process of the sample. 


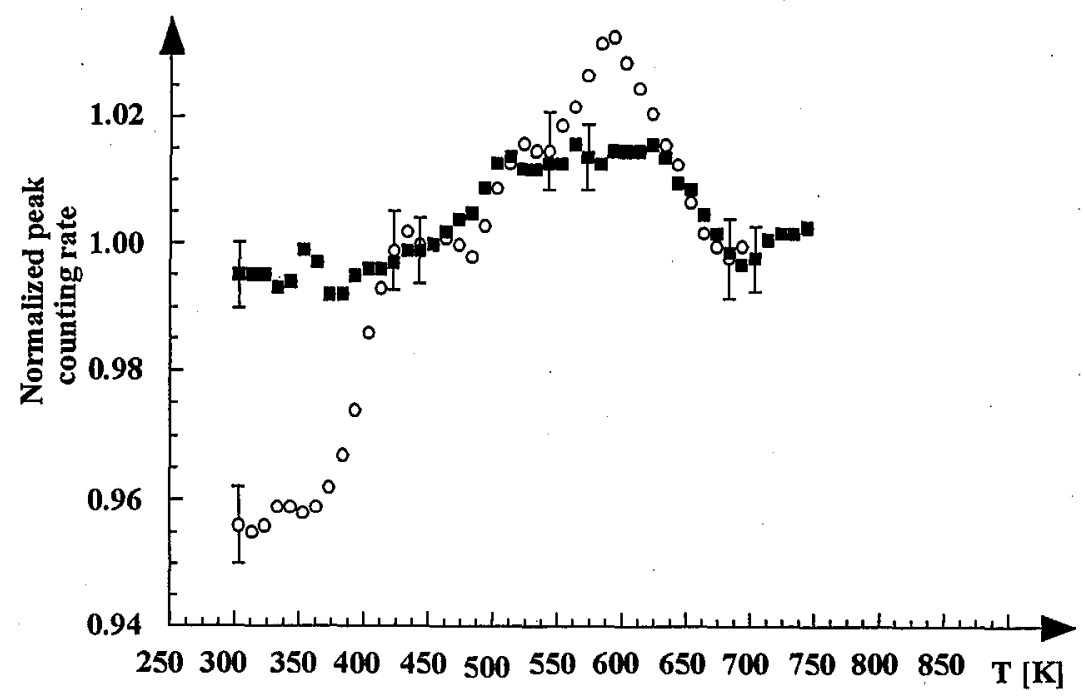

Fig. 2. Normalized peak counting rate as a function of the temperature for defected by cold rolling $\mathrm{Pd}-23 \% \mathrm{Cu}$ hydrogen-free sample (black squares), and for defected and hydrogenated sample (open circles).

Figure 2 shows the temperature dependence of the normalized coincidence peak counting rate for the defected by cold-rolling up to deformation degree of $50 \%$ hydrogen-free $\mathrm{PdCu}$ sample (black squares) and hydrogenated one (open circles) in the temperature interval from room temperature to $750 \mathrm{~K}$. One can separate four parts in the curve for hydrogen-free sample. The first part in the range of the temperature from room temperature to about $450 \mathrm{~K}$. In this interval of the temperature, saturation of the positron annihilation frin the trapped state already occurs [4]. These trap states probably are not vacancies that should be annealed in lower than room temperature. These traps may probably be vacancy clusters or voids and dislocations that are stable up to the temperature of about $380 \mathrm{~K}$. Some of the traps migrate and agglomerate to larger defects (the second part of the curve). These larger defects are stable up to the temperature of about $650 \mathrm{~K}$ (the third part of the curve). At higher temperatures recrystallization process of the sample starts to take course (the fourth part of the curve). After introducing hydrogen into a sample, hydrogen atoms may fill out some of the traps and form small hydrogen bubbles, which appear as a decrease in the first part of the curve in the temperature interval from room temperature to about $360 \mathrm{~K}$ (open circles). In higher temperatures hydrogen atoms release the traps and further run of the curve is the same as of the curve for hydrogen-free samples, but apart of the temperature. interval between $560 \mathrm{~K}$ and $640 \mathrm{~K}$. It is difficult to find reasonable explanation for the peak at the temperature of $575 \mathrm{~K}$ in the curve for hydrogenated sample. This peak seems to be related to more stable hydrogen-defect complexes.

The results of the positron lifetime measurements for the four samples are collected in Table. The mean lifetime $\tau_{1}$ represents the averaged value of the bulk lifetime and the lifetime due to the trapping of positrons at vacancies and 
TABLE

Positron lifetime values for the investigated $\mathrm{Pd}-23 \% \mathrm{atCu}$.

\begin{tabular}{l|c|c|c|c}
\hline \hline \multicolumn{1}{c|}{ Sample } & $\tau_{1}[\mathrm{ps}]$ & $I_{1}[\%]$ & $\tau_{2}[\mathrm{ps}]$ & $I_{2}[\%]$ \\
\hline Annealed, hydrogen free & $121 \pm 1$ & 97 & $341 \pm 1$ & 3 \\
Annealed and hydrogenated & $123 \pm 7$ & 80 & $231 \pm 20$ & 20 \\
Defected, hydrogen free & $143 \pm 5$ & 35 & $224 \pm 7$ & 65 \\
Defected and hydrogenated & $138 \pm 6$ & 14 & $187 \pm 9$ & 86
\end{tabular}

dislocations. Its value, for annealed hydrogen-free sample, equals to $121 \mathrm{ps}$ with the intensity $I_{1}=97 \%$. Apart from the component $\tau_{1}$, there is one component $\tau_{2}=341 \mathrm{ps}$ with the intensity $I_{2}=3 \%$ which may appear most probably on account of the source and will not be discussed. For the sample saturated with hydrogen, the value of component $\tau_{1}$ did not change, but the appearing component of $\tau_{2}$ with its value of $233 \mathrm{ps}$ and intensity of $20 \%$ is most probably connected with hydrogen-induced defects of voids type. In the case of defected hydrogen-free sample, the value $\tau_{1}=143 \mathrm{ps}$ with the intensity $I_{1}=35 \%$. The lifetime component $\tau_{2}=224 \mathrm{ps}$ with the intensity $I_{2}=65 \%$. Introduction of hydrogen into sample does not substantially affect the value of $\tau_{1}$ that equals $138 \mathrm{ps}$ whereas the intensity of $I_{1}$ decreases from $35 \%$ to $14 \%$. Changes in the lifetime and its intensity are in accordance with earlier discussion on the course of the temperature dependences.

\section{Conclusions}

Hydrogen atoms introduced into an annealed $\mathrm{Pd}-23 \%$ atCu alloy sample can form defects, probably hydrogen vacancy complexes type, that in higher temperatures are annealed or migrate to form more stable H-defect systems. These systems are stable up to the temperature of about $500 \mathrm{~K}$.

In the defected by cold-rolling and hydrogenated $\mathrm{Pd}-23 \%$ atCu sample, hydrogen can combine with defects like clusters, voids and dislocations, may fill out some of them and form small hydrogen bubbles.

\section{References}

[1] M. Hansen, K. Anderko, Constitution of Binary Alloys, McGraw-Hill, New York 1959.

[2] I.B. Condon, T. Schober, I. Nucl. Mat. 207, 1 (1993).

[3] B. Nielson, A. Van Vien, L.M. Caspers, W. Lourens, Cr. Trumpy, K. Peterson, in: Positron Annihilation, Eds. P.G. Coleman, S.C. Sharma, L.M. Diana, North-Holland, Amsterdam 1982.

[4] G. Dlubek, G. Brauer, O. Brümmer, L. Andrejtchev, P. Manfrass, Phys. Status. Solidi A 30, K37 (1975). 